\title{
Tyrosine kinase-targeting drugs-associated heart failure
}

\author{
N Gronich ${ }^{*}{ }^{1}$, I Lavi $^{1}$, O Barnett-Griness ${ }^{1}$, W Saliba ${ }^{1}$, D R Abernethy ${ }^{2}$ and G Rennert ${ }^{1,3}$ \\ ${ }^{1}$ Department of Community Medicine and Epidemiology, Lady Davis Carmel Medical Center, and Bruce Rappaport Faculty of \\ Medicine, Technion-Israel Institute of Technology, 7 Michal Street, Haifa 3436212, Israel; ${ }^{2}$ Drug Safety Group, Office of Clinical \\ Pharmacology, US Food and Drug Administration, 10903 New Hampshire Avenue, Silver Spring, MD 20993, USA and ${ }^{3}$ Office of \\ Chief Physician, Clalit Health Services, 101 Arlozorov Street, Tel Aviv 6209804, Israel
}

Background: The impact of cancer therapies on cardiac disease in the general adult cancer survivor population is largely unknown. Our objective was to evaluate which tyrosine kinase-targeting drugs are associated with greater risk for new-onset heart failure (HF).

Methods: A nested case-control analysis was conducted within a cohort of 27992 patients of Clalit Health Services, newly treated with a tyrosine kinase-targeting, and/or chemotherapeutic drug, for a malignant disease, between 1 January 2005 and 31 December 2012. Each new case of HF was matched to up to 30 controls from the cohort on calendar year of cohort entry, age, gender, and duration of follow-up. Main outcome measure was odds ratio (OR) with $95 \%$ confidence interval (Cl) of new-onset $\mathrm{HF}$.

Results: There were 936 incident cases of HF during 71742 person-years of follow-up. Trastuzumab (OR 1.90, 95\% Cl 1.46-2.49), cetuximab (OR 1.72, 1.10-2.69), panitumumab (OR 3.01, 1.02-8.85), and sunitinib (OR 3.39, 1.78-6.47) were associated with increased HF risk. Comorbidity independently associated with higher risk in a multivariable conditional regression model was diabetes mellitus, hypertension, chronic renal failure, ischaemic heart disease, valvular heart disease, arrhythmia, and smoking.

Conclusions: Trastuzumab, cetuximab, panitumumab, and sunitinib are associated with increased risk for new-onset HF.

Modern cancer treatment strategies have led to an improvement in the chances of survival. However, long-term side effects have emerged (Siegel et al, 2012). The incidence of cancer treatmentinduced cardiac injury varies widely, depending on the specific cancer therapy used, duration of therapy, and underlying patient comorbidity. The impact of cancer therapies on cardiac disease in the general adult cancer survivor population is largely unknown.

Conventional chemotherapeutics can induce permanent myocardial cell injury, leading to acute or chronic left ventricular dysfunction (Yeh and Bickford, 2009). Tyrosine kinase-targeting drugs, gaining central role in the treatment of haematological malignancies and solid tumours are, in general, less toxic than conventional chemotherapy and are usually well tolerated, but might cause severe adverse effects including heart failure (HF) (Krause and Van Etten, 2005; Eschenhagen et al 2011; Moslehi,
2016). Frequency of HF and the tyrosine kinase-targeting drugs that are associated with greater risk are not well described. As this adverse effect was unexpected, first clinical trials did not include cardiac end points and later trials excluded patients with cardiac diseases or risk factors. Except for data on trastuzumab, data on cardiotoxicity of the rest of the tyrosine kinase-targeting drugs are sparse and there is not much originating from real-world use, where patients treated with tyrosine kinase-targeting drugs frequently are old and suffer multiple diseases (Moslehi, 2016). There are two types of tyrosine kinase-targeting drugs: small molecules that inhibit the activity of the intracellular kinases; and humanised monoclonal antibodies directed against receptor tyrosine kinases or their ligands (Supplementary Table 1). There is a need to identify which are the tyrosine kinase-targeting drugs that are associated with greater risk for $\mathrm{HF}$, and what the

*Correspondence: Dr N Gronich; E-mail: gronichn@clalit.org.il

Received 30 October 2016; revised 17 January 2017; accepted 23 February 2017; published online 11 April 2017

(C) 2017 Cancer Research UK. All rights reserved 0007-0920/17 
contributing factors are for developing the adverse effect, to allow a better decision process when there is a choice between different drugs (Moslehi, 2016). Tyrosine kinase targeting drug-associated cardiomyopathy is thought to have a different biological mechanism than chemotherapy-associated cardiomyopathy, and to be many times reversible (Ewer and Lippman, 2005a; Jones et al, 2009). It is, thus, important to identify patients who belong to higher-risk groups, for a closer follow-up and early diagnosis of the adverse effect.

We were able to evaluate and compare among 13 tyrosine kinase-targeting drugs that were in use during 8 years in one health system, controlling for comorbidity and co-medications, and for chemotherapy use.

\section{MATERIALS AND METHODS}

We conducted a cohort study with a nested case-control analysis using Clalit Health Services (CHS) database. Clalit Health Services is the largest health services provider in Israel providing medical care to 4.2 million, which is more than half of the Israeli population. All clinical and administrative data of CHS are centrally computerised since 1998 to produce electronic medical records that include all patients' diagnoses from primary-care physician and specialty clinics in the community (coded using the International Statistical Classification of Diseases, Ninth Revision (ICD-9)), anthropometric and lifestyle variables such as body mass index (BMI), and smoking, full hospitalisation records, radiology, laboratory, and pharmacy data of filled prescriptions. This database has been formerly described and is valid and reliable (Matok et al, 2009; Gronich et al, 2011). A chronic disease registry is compiled from these data sources. Diagnoses are captured in the registry by diagnosis-specific algorithms, using both code reading (e.g. ICD-9 and International Classification of Primary Care) and text reading. A record is kept of the sources and dates used to establish the diagnosis, with the earliest recorded date being considered the starting date.

We selected all patients newly diagnosed with any type of malignancy, excluding non-melanoma skin cancer (ICD-9 codes: 140-172 and 174-208), between 1 January 2005 and 31 December 2012, and no past diagnosis of a malignant disease. We used drugdispensing data from CHS pharmacies to select, within these patients, all patients who have begun treatment with a tyrosine kinase-targeting drug, anti-CD20 (rituximab), and/or a chemotherapeutic drug between 1 January 2005 and 31 December 2012. These patients formed the study cohort. The date of entry to the cohort was the date of first prescription of a chemotherapeutic, anti-CD20, or a tyrosine kinase targeting drug. We excluded patients who did not have at least 6 months of medical history in CHS before entry to the cohort. We also excluded patients with a history of dialysis due to end-stage renal disease and patients with history of HF or cardiomyopathy at cohort entry. We followed the patients until 31 December 2012, or until a new diagnosis of HF or cardiomyopathy, death, or end of registration in CHS, whichever came first.

Selection of cases and controls. A nested case-control analysis (Richardson, 2004; Schneeweiss and Suissa, 2012; Filion et al, 2016) was conducted within the cohort. We chose the nested casecontrol approach because of time-varying nature of drug used, and the long duration of follow-up. Compared with a full cohort approach using a survival analysis with time-dependent variables, a nested case-control analysis is computationally more efficient, while providing an unbiased estimate of the rate ratio in the cohort. Cases were defined by an emergence of a diagnosis of $\mathrm{HF}$ or cardiomyopathy during follow-up (ICD-9 codes: 398.91; 402.01; $402.11 ; 402.91 ; 404.01 ; 404.03 ; 404.11 ; 404.13 ; 404.91 ; 404.93$;
$416.9 ; 425 ; 425.0 ; 425.2 ; 425.3 ; 425.4 ; 425.5 ; 425.7 ; 425.8 ; 425.9$; $428 ; 428.0 ; 428.1 ; 428.2 ; 428.20 ; 428.22 ; 428.23 ; 428.30 ; 428.31$; $428.32 ; 428.33 ; 428.40 ; 428.42 ; 428.43 ; 428.9$; and 514$)$. The first date of diagnosis of HF from hospitalisation/primary-care physician/specialty clinic in the community defined the index date. Because HF following antineoplastic treatment can be an acute or late effect we considered a new case diagnosed since the first day of follow-up with no latency time window.

For each case, up to 30 controls were randomly selected from the cohort using risk set sampling with replacement by SAS version 9.3 (SAS Institute Inc., Cary, NC, USA), matched by calendar year of cohort entry, age \pm 2 years, gender, and duration of follow-up. Controls had to be at risk of the event on the case's index date. Thus, index dates of the controls were the dates corresponding to the same follow-up days as their matched cases such that follow-up days were equal.

Use of tyrosine kinase-targeting drugs and potential confounders. We obtained information on all tyrosine kinase-targeting drugs, anti-CD20, and chemotherapeutic antineoplastic treatment dispensed to each patient in the cohort from cohort entry to index date. At least one dispensed dose defined exposure.

Potential confounders data acquired: BMI, weight, height, and smoking status last recorded before the index date, alcohol abuse, and drug abuse; history of chronic diseases, known to be associated with HF, diagnosed before cohort entry to avoid adjusting for factors affected by exposure: diabetes mellitus, hypertension, hyperlipidaemia, ischaemic heart disease, peripheral vascular disease, carotid artery disease, atrial fibrillation, other arrhythmias, valvular heart disease, chronic renal failure, and cirrhosis; medications known to be potentially associated with HF (Maxwell and Jenkins, 2011), dispensed in the last year before the index date; other antineoplastic and immunomodulating agents dispensed in the last year before the index date; and radiotherapy (all fields) during follow-up until index date. Antiarrhythmic drugs used were assessed before cohort entry because these drugs, in addition to being a potential cause for HF, can also be on the causal path between exposure and HF (when prescribed for an arrhythmia caused by exposure to chemotherapy or to a tyrosine kinase-targeting drug). We also obtained number of days of hospital admissions before cohort entry as a measure of severity of chronic diseases. Drugs and potential confounders with total frequencies of $<0.1 \%$ were not included in the analyses.

Statistical analysis. We calculated the incidence rate by dividing the total number of cases by the total number of person-years of follow-up in the cohort, with $95 \%$ confidence intervals (CIs) based on Poisson distribution. To account for the nested case-control sampling, we used multivariate conditional logistic regression to estimate odds ratio (OR) and corresponding 95\% CIs for the association between each tyrosine kinase-targeting/anti-CD20 drug and the risk of incident HF. This model included a binary indicator variable for each tyrosine kinase-targeting/anti-CD20 drug. Odds ratios were adjusted for comorbidity and drugs potentially associated with $\mathrm{HF}$, with categorical terms (yes $v s$ no) for each of the following variables: diabetes mellitus; hypertension; hyperlipidaemia; ischaemic heart disease; peripheral vascular disease; carotid artery disease; atrial fibrillation; other arrhythmias; valvular heart disease; chronic renal failure; cirrhosis; obesity; smoking; alcohol abuse; drug abuse; treatment with class I or III antiarrhythmics, thiazolidinediones, systemic corticosteroids, nonsteroidal anti-inflammatory drugs, systemic antimycotics imidazole and triazole derivatives, tumour necrosis factor alpha inhibitors, everolimus (low dose), temsirolimus, clozapine, colony-stimulating factors, gonadotropin-releasing hormone analogues, radiotherapy, and the chemotherapy groups including nitrogen mustard analogues, other alkylating agents, folic acid analogues, purine 
analogues, pyrimidine analogues, vinca alkaloids and analogues, etoposide, taxanes, anthracyclines and related substances (doxorubicin, daunorubicine, epirubicin, idarubicin, and mitoxantrone), bleomycin, mitomycin, platinum compounds, procarbazine, other chemotherapeutics (estramustine, tretinoin, mitotane, pegaspargase, arsenic trioxide, anagrelide, and visemodegib), everolimus (high dose), hydroxycarbamide, bortezomib, topotecan, and irinotecan; and a continuous variable for hospital admission duration.

Next, we divided tyrosine kinase-targeting drugs to two groups: monoclonal antibodies group and the small molecules group, and included these two groups in the multivariable logistic regression analysis to allow evaluation of the type of molecule to HF mechanism, adjusted to all variables described above.

We also grouped the drugs into four groups defined by their main targeted molecule: EGFR-targeted therapies, including ErbB1 and ErbB2 (cetuximab, panitumumab, gefitinib, erlotinib, trastuzumab, and lapatinib); VEGFR-targeted drugs (bevacizumab, sorafenib, sunitinib, and pazopanib); BCR-ABL-targeted drugs (imatinib, nilotinib, and dasatinib); and the anti-CD20 drug (rituximab), to assess a probable role of the targeted molecule inhibition in myocardial damage, and performed a multivariable

Table 1. Clinical characteristics and systemic chemotherapy treatment of cases with heart failure and matched controls in a cohort of patients with malignant diseases

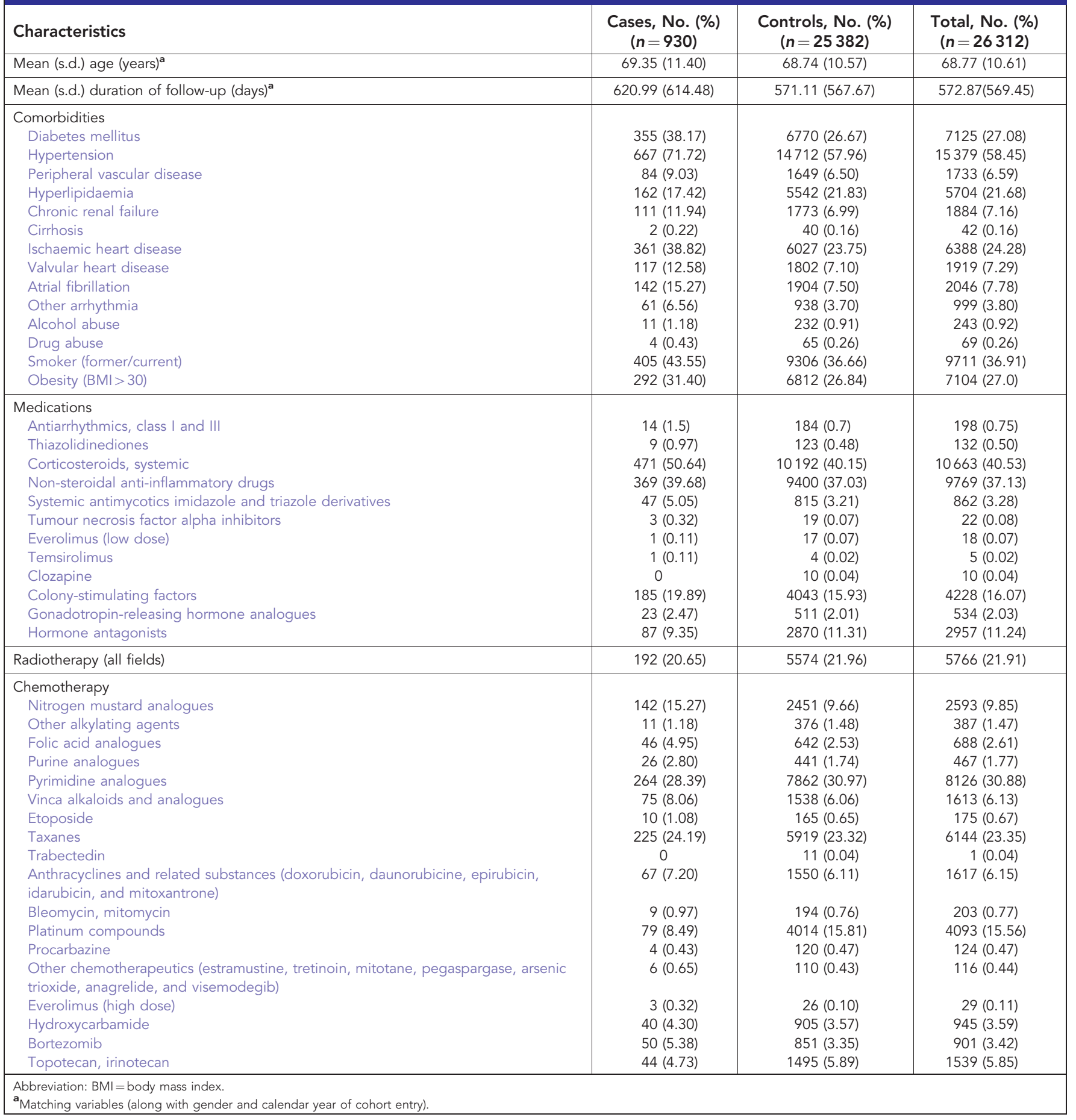


logistic regression analysis adjusted for all potential confounders described above.

As a sensitivity analysis we stratified the cohort by cancer type and fitted a single model to the entire data set adjusting for all potential confounders described above. We also performed a separate multivariable logistic regression model for each cancer type with 100 cases or more and their matched controls, adjusting for all potential confounders described above, with frequencies of more than $1 \%$.

In another sensitivity analysis we considered only patients who did not receive any chemotherapy drug thought to cause cardiomyopathy, and patients who did receive at least one chemotherapy drug known to cause cardiomyopathy by stratified multivariable logistic regression analysis.

We also performed a sensitivity analysis by multivariable logistic regression analysis with stratification to patients with and without major risk factors or pre-existing heart disease when they were diagnosed with their cancer.

All $P$-values were two-tailed and $P<0.05$ was considered significant. Analyses were done with SPSS version 21 (IBM Corporation, Armonk, New York, NY, USA).

\section{RESULTS}

A total of 120440 CHS patients were newly diagnosed with any type of malignancy, between 1 January 2005 and 31 December 2012. In all, 30902 out of 120440 met the inclusion criteria of receiving a tyrosine kinase-targeting drug and/or systemic chemotherapeutic antineoplastic treatment. In all, 221 patients who did not have at least 6 months of medical history in CHS before cohort entry date were excluded. A total of 1282 patients requiring dialysis due to ESRD were excluded, as well as 1407 patients that carried a diagnosis of $\mathrm{HF}$ or cardiomyopathy before cohort entry. The study cohort included 27992 patients.

Within the cohort, we identified 936 cases that were newly diagnosed with HF during 71742 person-years of follow-up, yielding an overall rate of 13 cases of HF per 1000 person-years (95\% CI 12.22-13.91). A total of 930 cases were successfully matched to 25382 controls by calendar year of cohort entry, age \pm 2 years, gender, and duration of follow-up. For six cases controls could not be matched and thus were not included in further analyses. Eighty-seven per cent of cases were matched to 30 controls; for $94.5 \%$ of cases at least 20 controls were matched; at least 10 controls were matched to $99 \%$ of cases.

Characteristics of cases and matched controls and their chemotherapeutic drug use are described in Table 1. Diagnoses of malignant diseases of cases and matched controls are listed in Supplementary Table 2. Number of cases and matched control patients, mean age, and main malignant disease diagnosed for each tyrosine kinase-targeting drug users' group are listed in Supplementary Table 3.

In a multivariable analysis of specific associations with each tyrosine kinase-targeting drug, adjusting for comorbidity and drugs potentially associated with $\mathrm{HF}$, trastuzumab (OR 1.90), cetuximab

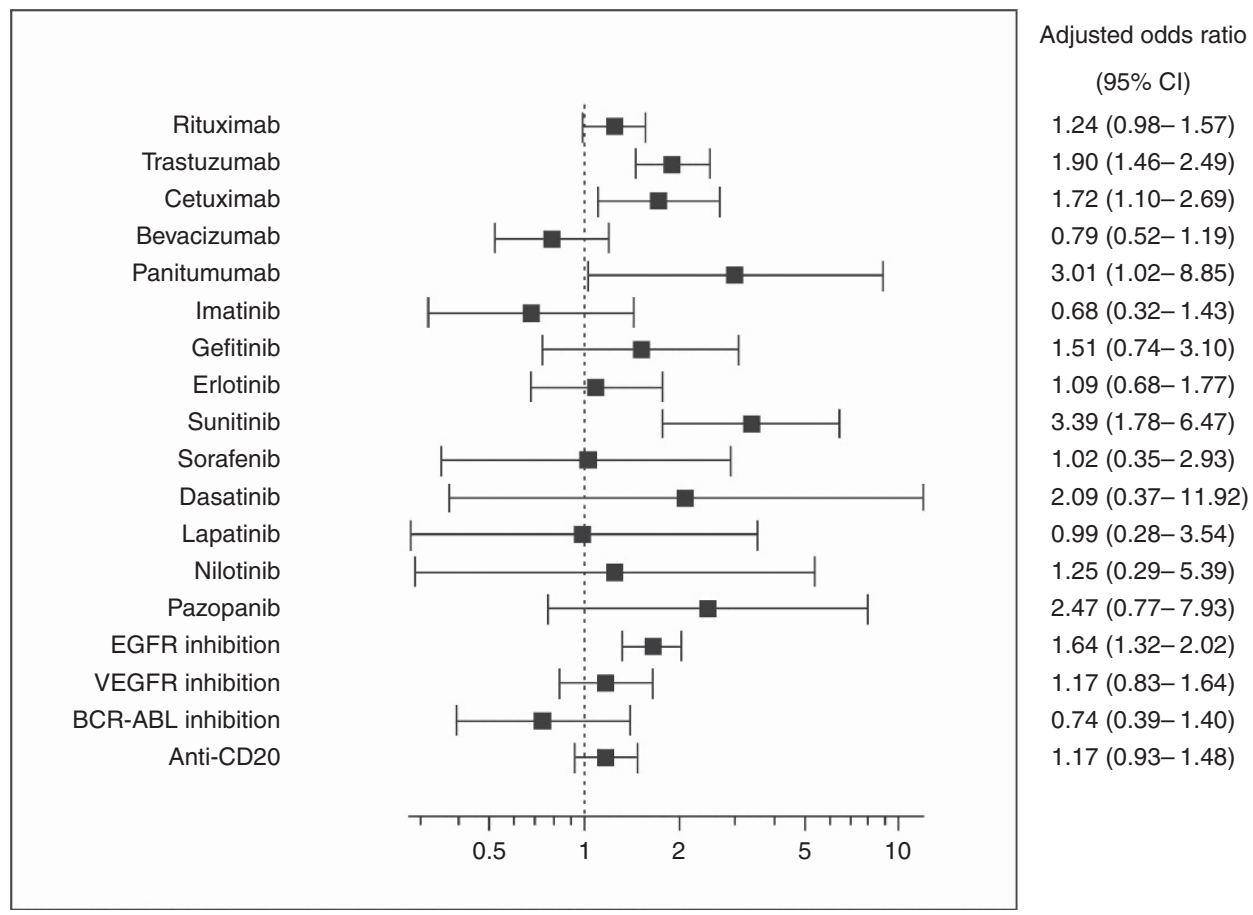

Figure 1. Forest plot showing the risk of developing new-onset heart failure associated with tyrosine kinase-targeting and anti-CD20 drugs. Nested case-control study ( $N=26312)$ matched by age, gender, duration of follow-up, and calendar year of cohort entry, within a cohort of patients newly diagnosed with malignant diseases and treated systemically. Multivariable model adjusted for diabetes mellitus, hypertension, hyperlipidaemia, ischaemic heart disease, peripheral vascular disease, carotid artery disease, atrial fibrillation, other arrhythmias, valvular heart disease, chronic renal failure, cirrhosis, obesity, smoking, alcohol abuse, drug abuse, class I and III antiarrhythmics, thiazolidinediones, systemic corticosteroids, non-steroidal anti-inflammatory drugs, systemic antimycotics imidazole and triazole derivatives, tumour necrosis factor alpha inhibitors, everolimus, temsirolimus, clozapine, colony-stimulating factors, gonadotropin-releasing hormone analogues, radiotherapy, the chemotherapy groups including: nitrogen mustard analogues, other alkylating agents, folic acid analogues, purine analogues, pyrimidine analogues, vinca alkaloids and analogues, etoposide, taxanes, anthracyclines and related substances, bleomycin, mitomycin, platinum compounds, procarbazine, other chemotherapeutics (estramustine, tretinoin, mitotane, pegaspargase, arsenic trioxide, anagrelide, and visemodegib), hydroxycarbamide, bortezomib, topotecan, and irinotecan, and hospital admission duration. 
(OR 1.72), panitumumab (OR 3.01), and sunitinib (OR 3.39) were associated with a statistically significant increased risk of new-onset HF (Supplementary Table 4; Figure 1). Median time was 520, 242, 168 , and 291 days, respectively, from beginning of trastuzumab, cetuximab, panitumumab, and sunitinib treatment, respectively, until HF was diagnosed. A sensitivity analysis in which drugs and potential confounders with frequency of $<0.1 \%$ in the entire cohort were included in the model did not change the main results.

In an analysis by the targeted molecule, EGFR inhibition was associated with increased HF risk. VEGFR inhibition, BCR-ABLtargeted inhibition, and the anti-CD20 mechanisms were not associated with increased HF risk (Figure 1). In an analysis of the type of molecule, monoclonal antibodies as a group were associated with increased HF risk (OR 1.33, 95\% CI 1.12-1.57); small molecules group risk did not reach statistical significance (OR 1.28, 95\% CI 0.96-1.71).

In a sensitivity analysis, stratifying by cancer types in a multivariable conditional regression model, magnitude of associated effects of trastuzumab, cetuximab, and sunitinib maintained with ORs 2.27 (1.67-3.08), 1.69 (0.92-3.11), and 3.80 (1.55-9.29), respectively. The magnitude of effect associated with panitumumab decreased with OR $1.93(0.51-7.27)$. In a sensitivity analysis applying a multivariable conditional regression model of breast cancer patients only $(N=2318)$ trastuzumab was associated with increased HF risk (OR 1.86, 1.30-2.66); cetuximab was associated with increased risk in a multivariable conditional regression model of colon cancer patients only $(N=1162$; OR 2.59, 1.09-6.12).

In a sensitivity analysis using conditional multivariable logistic regression stratifying to patients who were exposed to chemotherapies known to be associated with HF (nitrogen mustard analogues, pyrimidine analogues, anthracyclines and related substances, etoposide, taxanes, mitomycin, and bleomycin), and patients not exposed to at least one of these chemotherapies, magnitude of effects of association between new-onset HF, and trastuzumab and cetuximab increased: ORs $6.37(1.41-28.74)$ and 12.96 (1.26-133.20) respectively, in patients not receiving chemotherapies with known risk, and 2.05 (1.52-2.77), 2.54 (1.29-4.99), and 3.91 (1.11-13.81) for trastuzumab, cetuximab, and panitumumab, respectively, in patients receiving chemotherapy with known risk. Sunitinib association could not be evaluated in this analysis because of the paucity of sunitinib-treated patients in the chemotherapy-exposed groups.

In a sensitivity analysis, using stratified conditional multivariable logistic regression trastuzumab OR 3.61 (1.88-6.93) and rituximab OR 3.57 (1.30-9.76) were associated with increased risk for new-onset HF in patients without risk factors for HF or preexisting heart disease. In patients carrying at least one risk factor for $\mathrm{HF}$ or pre-existing heart disease while entering the study (including diabetes mellitus, hypertension, smoking, ischaemic heart disease, valvular heart disease, and arrhythmia), tratzuzumab, cetuximab, panitumumab, and sunitinb were associated with increased HF risk, ORs of 1.83 (1.34-2.50), 1.90 (1.21-2.98), 3.89 (1.33-11.33), and 3.35 (1.76-6.35), respectively.

\section{DISCUSSION}

Our large population-based study was designed to assess the association between tyrosine kinase-targeting drugs and HF in real-world setting. In this cohort study of patients that were treated systemically for malignant diseases we have found that trastuzumab, cetuximab, panitumumab, and sunitinib were associated with new-onset HF.

Of the drugs evaluated here, the cardiotoxicity of the monoclonal antibody HER-2 (EGFR2 or ErbB2) inhibitor trastuzumab has been the most well characterised with RR of
3.19 (95\% CI, 2.03-5.02) and an increased risk with concomitant or sequential chemotherapy treatment (Seidman et al, 2002; Perez et al, 2008). Clinical risk factors described were older age, coronary artery disease, and hypertension (Ewer et al, 2005b; ChavezMacGregor et al, 2013). Conditional mutant mice carrying a cardiac-restricted deletion of ErbB2 (HER-2) developed cardiac chamber dilation, wall thickening, and decreased contractility (Crone et al, 2002; Ozcelik et al, 2002). Patients who have been developing cardiotoxicity while receiving trastuzumab generally improved on removal of the agent (Goldhar et al, 2015). Lapatinib seemed to be associated with lower risk but none of the trials has directly compared trastuzumab and lapatinib (Johnston et al, 2009; Blackwell et al, 2010). The monoclonal antibody EGFR (ErbB1) inhibitor cetuximab had been reported to rarely cause HF (Vermorken et al, 2008), and panitumumab had not been previously associated with HF (Douillard et al, 2014), but in a recent meta-analysis of EGFR (ErbB1) inhibitors in upper gastrointestinal tract cancers anti-EGFR treatment was associated with higher rates of cardiac events (Li et al, 2015). Chronic erlotinib treatment has been shown recently to induce mild-tomoderate cardiac dysfunction in rats (Mak et al, 2015). In our large population analysis with up to 8 years of follow-up trastuzumab, cetuximab, and panitumumab, all inhibitors of EGFR signalling, increased HF risk. EGFR signalling is required for growth, repair, and survival of cardiomyocytes. EGFR signalling is thought, thus, to be essential for the prevention of dilated cardiomyopathy. The mechanism of cardiotoxicity is thought to be different from that of chemotherapy-related cardiotoxicity, in part, demonstrated by the absence of cardiac ultrastructural changes (Ewer et al, 2005b).

In our analysis VEGFR inhibition, as a group, was not associated with increased HF risk. Individually, the small molecule, sunitinib, was associated with a reduction in LVEF, as has been shown in other studies (Chu et al, 2007; Ewer et al, 2014). Nevertheless, it was not exhibited in a randomised trial that compared sunitinib to interferon alfa (Motzer et al, 2007). Similar to our results, sorafenib had not been associated with HF (Escudier et al, 2009). Bevacizumab, a monoclonal antibody that binds VEGF, had not been associated with clinically significant increased HF risk as has been shown also in our analysis, but a decreased LVEF had been demonstrated previously in co-treatment with chemotherapy (McArthur et al, 2011). VEGF induces angiogenesis, by causing endothelial cells proliferation. Bevacizumab, sunitinib, and sorafenib, thus, have direct effects on the vasculature. They result in hypertension by causing endothelial cell dysfunction. Sunitinib is unique by also destroying pericytes, which wrap around blood vessels and are essential for blood vessel formation and maintenance. Sunitinib might also cause HF by an off-target effect through direct cardiomyocyte mitochondrial damage, inhibition of the compensatory upregulation of AMPK, and cytochrome-C-induced apoptosis (Hasinoff et al, 2008; Kerkelä et al, 2009; Chintalgattu et al, 2013).

The small-molecule BCR-ABL inhibitors imatinib and nilotininb had not been associated previously with increased HF risk (O'Brien et al, 2003; Kim et al, 2012; Larson et al, 2012), although Kerkelä et al (2006) presented a thorough investigation into the myocardial pathological changes in patients with HF who were treated with imatinib. A modest increased risk had been proposed with dasatinib (Brave et al, 2008), but did not reach statistical significance in our study. Similar to our data, rituximab, an anti-CD20 monoclonal antibody, has not been associated with significant HF risk.

The use of certain chemotherapeutics is known to be associated with cumulative dose-dependent cardiomyopathy, carrying increased mortality risk (Clark et al, 2016). In the British Childhood Cancer Survivor Study of late mortality after childhood cancer within a cohort of 34489 five-year survivors, circulatory causes accounted for the largest number of deaths due to non- 
neoplastic causes. Survivors were 3.8 times as at risk of death due to circulatory causes than expected from the general population (Fidler et al, 2016). Except for the well-described anthracyclineassociated HF, acute HF had been associated with the nitrogen mustard analogues cyclophosphamide (in 7-28\% of patients) and ifosfamide (in 17\% of patients; Gottdiener et al, 1981; Goldberg et al, 1986; Braverman et al, 1991; Quezado et al, 1993). The incidence of HF associated with the taxane, docetaxel, ranges from 2.3 to $8 \%$ (Martin et al, 2005; Marty et al, 2005), and a growing number of case reports recognise cardiomyopathy associated with 5-FU treatment also (Stewart et al, 2010; Radhakrishnan and Bakhshi, 2011). Anthracyclines, alkylating agents, antimetabolites, and the taxanes, in addition to targeted therapies, have been identified by the American Heart Association in a recently published list of medications that might cause or exacerbate HF (Page et al, 2016). Our multivariable analysis present similar results of independent risk associated with various chemotherapeutics. In our analysis, anthracyclines were not associated with an increased cardiotoxicity effect. Awareness of physicians to anthracycline cardiotoxicity might have led to careful selection of patients to be treated with this class of drugs, and to the restriction of cumulative doses. To explore this, we have looked at differences between patients who were treated, and those not treated with anthracyclines and found fewer comorbidities in the anthracycline-treated group, as well as a higher rate of obesity (thus larger body surface area; Supplementary Table 5). Also, median anthracycline cumulative dose used in our cohort was $400 \mathrm{mg}$ (mean $424.9 \mathrm{mg}$ ) and interquartile range $(25-75 \%)$ of $220-500 \mathrm{mg}$, while the limit above which cardiotoxicity is known to increase is $400 \mathrm{mg} \mathrm{m}^{-2}$ for doxorubicin and $900 \mathrm{mg} \mathrm{m}^{-2}$ for epirubicin (the two mostly used anthracyclines in our patients). In addition, the early-onset anthracycline cardimyopathy (during therapy or within the first year after treatment) occurs in $1.6-2.1 \%$ of patients, while lateoccurring chronic progressive cardiotoxicity might not become evident until 10-20 years after the first dose of cancer treatment (Yeh and Bickford, 2009), and thus would not been captured in our study.

Our study has some limitations. We were not able to assess cardiac function imaging results. Our characterisation of HF was based on ICD-9 codes. However, this was probably an underestimation of cardiac dysfunction. In the N9831 adjuvant breast cancer trial, for example, incidence of asymptomatic left ventricular ejection fraction was $8-10 \%$ (Perez et al, 2008). It has also been shown in studies of HF coding in similar data sets that a registered diagnosis of $\mathrm{HF}$ carries a very high specificity and lower sensitivity (Khand et al, 2005; Lee et al, 2005; Kümler et al, 2008). Second, it was beyond the scope of our study to assess more than 1000 different combinations of tyrosine kinase-targeting drugs with specific chemotherapeutics. Third, due to small number of users of the newer tyrosine kinase-targeting drugs and shorter follow-up time, our study could not evaluate these drugs with the same confidence. As shown in Supplementary Table 3 total number of users of panitumumab, dasatinib, lapatinib, nilotinib, and pazopanib were $57,27,28,56$, and 37 , respectively. Thus, random error could have occurred in the analysis of these drugs individually due to small sample sizes. Specifically, our finding on the excess risk associated with panitumumab should be interpreted with caution. Fourth, we could not acquire data on the effect of specific radiation fields on HF. Finally, patients receiving drugs known to be associated with HF, such as trastuzumab, might have been under more intensive surveillance, and thus clinicians' overestimation of HF might have occurred with specific drugs.

Our study has a number of strengths. With 27992 patients and 71742 person-years of observation, we had the statistical power to robustly assess this important drug safety issue. By studying realworld data we were able to evaluate association with side effects that might have become apparent only in the older and sicker population that was generally excluded from clinical trials. Our ability to assess all tyrosine kinase-targeting drugs that were in use during the study period concomitantly was strength of our report. We used rigorous matching and statistical adjustment to minimise residual confounding. In addition, by matching the controls to cases by calendar year, we reduced a bias associated with heightened awareness of physicians to tyrosine kinase-targeting drug-related adverse effects in later years.

\section{CONCLUSIONS}

In this large population-based cohort study we are showing that out of 13 tyrosine kinase-targeting drugs, trastuzumab, cetuximab, panitumumab, and sunitinib are associated with increased risk to develop HF. The survival benefit that tyrosine kinase-targeting drugs have provided is clear. However, when there is a choice between different tyrosine kinase-targeting drugs with different risks, or when other risk factors for HF are present and the benefit with treatment is less clear, then clinical considerations should take into account the risk associated with treatment. A closer follow-up on trastuzumab-, cetuximab-, panitumumab-, and sunitinibtreated patients for early diagnosis of cardiomyopathy might be suggested, especially for patients who carry other clinical risk factors. Further research should evaluate HF risk associated with dasatinib, lapatinib, nilotinib, and pazopanib, as well as newly appearing tyrosine kinase-targeting drugs.

\section{CONFLICT OF INTEREST}

The authors declare no conflict of interest.

\section{REFERENCES}

Blackwell KL, Burstein HJ, Storniolo AM, Rugo H, Sledge G, Koehler M, Ellis C, Casey M, Vukelja S, Bischoff J, Baselga J, O’Shaughnessy J (2010) Randomized study of Lapatinib alone or in combination with trastuzumab in women with ErbB2-positive, trastuzumab-refractory metastatic breast cancer. J Clin Oncol 28: 1124-1130.

Brave M, Goodman V, Kaminskas E, Farrell A, Timmer W, Pope S, Harapanhalli R, Saber H, Morse D, Bullock J, Men A, Noory C, Ramchandani R, Kenna L, Booth B, Gobburu J, Jiang X, Sridhara R, Justice R, Pazdur R (2008) Sprycel for chronic myeloid leukemia and Philadelphia chromosome-positive acute lymphoblastic leukemia resistant to or intolerant of imatinib mesylate. Clin Cancer Res 14: 352-359.

Braverman AC, Antin JH, Plappert MT, Cook EF, Lee RT (1991) Cyclophosphamide cardiotoxicity in bone marrow transplantation: a prospective evaluation of new dosing regimens. J Clin Oncol 9: 1215-1223.

Chavez-MacGregor M, Zhang N, Buchholz TA, Zhang Y, Niu J, Elting L, Smith BD, Hortobagyi GN, Giordano SH (2013) Trastuzumab-related cardiotoxicity among older patients with breast cancer. J Clin Oncol 31: $4222-4228$.

Chintalgattu V, Rees ML, Culver JC, Goel A, Jiffar T, Zhang J, Dunner Jr K, Pati S, Bankson JA, Pasqualini R, Arap W, Bryan NS, Taegtmeyer H, Langley RR, Yao H, Kupferman ME, Entman ML, Dickinson ME, Khakoo AY (2013) Coronary microvascular pericytes are the cellular target of sunitinib malate-induced cardiotoxicity. Sci Transl Med 5: 187 ra69.

Chu TF, Rupnick MA, Kerkela R, Dallabrida SM, Zurakowski D, Nguyen L, Woulfe K, Pravda E, Cassiola F, Desai J, George S, Morgan JA, Harris DM, Ismail NS, Chen JH, Schoen FJ, Van den Abbeele AD, Demetri GD, Force T, Chen MH (2007) Cardiotoxicity associated with tyrosine kinase inhibitor sunitinib. Lancet 370: 2011-2019.

Clark RA, Berry NM, Chowdhury MH, McCarthy AL, Ullah S, Versace VL, Atherton JJ, Koczwara B, Roder D (2016) Heart failure following cancer treatment characteristics, survival and mortality of a linked health data analysis. Intern Med J 46: 1297-1306. 
Crone SA, Zhao YY, Fan L, Gu Y, Minamisawa S, Liu Y, Peterson KL, Chen J, Kahn R, Condorelli G, Ross Jr J, Chien KR, Lee KF (2002) ErbB2 is essential in the prevention of dilated cardiomyopathy. Nat Med 8: 459-465.

Douillard JY, Siena S, Cassidy J, Tabernero J, Burkes R, Barugel M, Humblet Y, Bodoky G, Cunningham D, Jassem J, Rivera F, Kocákova I, Ruff P, Błasińska-Morawiec M, Smakal M, Canon JL, Rother M, Oliner KS, Tian Y, Xu F, Sidhu R (2014) Final results from PRIME: randomized phase 3 study of panitumumab with FOLFOX4 for first-line treatment of metastatic colorectal cancer. Ann Oncol 25: 1346-1355.

Eschenhagen T, Force T, Ewer MS, de Keulenaer GW, Suter TM, Anker SD, Avkiran M, de Azambuja E, Balligand JL, Brutsaert DL, Condorelli G, Hansen A, Heymans S, Hill JA, Hirsch E, Hilfiker-Kleiner D, Janssens S, de Jong S, Neubauer G, Pieske B, Ponikowski P, Pirmohamed M, Rauchhaus M, Sawyer D, Sugden PH, Wojta J, Zannad F, Shah AM (2011) Cardiovascular side effects of cancer therapies: a position statement from the Heart Failure Association of the European Society of Cardiology. Eur J Heart Fail 13: 1-10.

Escudier B, Eisen T, Stadler WM, Szczylik C, Oudard S, Staehler M, Negrier S, Chevreau C, Desai AA, Rolland F, Demkow T, Hutson TE, Gore M, Anderson S, Hofilena G, Shan M, Pena C, Lathia C, Bukowski RM (2009) Sorafenib for treatment of renal cell carcinoma: final efficacy and safety results of the phase III treatment approaches in renal cancer global evaluation trial. J Clin Oncol 27: 3312-3318.

Ewer MS, Suter TM, Lenihan DJ, Niculescu L, Breazna A, Demetri GD, Motzer RJ (2014) Cardiovascular events among 1090 cancer patients treated with sunitinib, interferon, or placebo: a comprehensive adjudicated database analysis demonstrating clinically meaningful reversibility of cardiac events. Eur J Cancer 50: 2162-2170.

Ewer MS, Lippman SM (2005a) Type II chemotherapy-related cardiac dysfunction: time to recognize a new entity. J Clin Oncol 23: 2900-2902.

Ewer MS, Vooletich MT, Durand JB, Woods ML, Davis JR, Valero V, Lenihan DJ (2005b) Reversibility of trastuzumab-related cardiotoxicity: new insights based on clinical course and response to medical treatment. $J$ Clin Oncol 23: 7820-7826.

Fidler MM, Reulen RC, Winter DL, Kelly J, Jenkinson HC, Skinner R, Frobisher C, Hawkins MM, British Childhood Cancer Survivor Study Steering Group (2016) Long term cause specific mortality among 34489 five year survivors of childhood cancer in Great Britain: population based cohort study. BMJ 354: i4351.

Filion KB, Azoulay L, Platt RW, Dahl M, Dormuth CR, Clemens KK, Hu N, Paterson JM, Targownik L, Turin TC, Udell JA, Ernst P, CNODES Investigators (2016) A multicenter observational study of incretin-based drugs and heart failure. $N$ Engl J Med 374: 1145-1154.

Goldberg MA, Antin JH, Guinan EC, Rappeport JM (1986) Cyclophosphamide cardiotoxicity: an analysis of dosing as a risk factor. Blood 68: 1114-1118.

Goldhar HA, Yan AT, Ko DT, Earle CC, Tomlinson GA, Trudeau ME, Krahn MD, Krzyzanowska MK, Pal RS, Brezden-Masley C, Gavura S, Lien K, Chan KK (2015) The temporal risk of heart failure associated with adjuvant trastuzumab in breast cancer patients: a population study. J Natl Cancer Inst 108: djv301.

Gottdiener JS, Appelbaum FR, Ferrans VJ, Deisseroth A, Ziegler J (1981) Cardiotoxicity associated with high-dose cyclophosphamide therapy. Arch Intern Med 141: 758-763.

Gronich N, Lavi I, Rennert G (2011) Higher risk of venous thrombosis associated with drospirenone-containing oral contraceptives: a population-based cohort study. CMAJ 183: E1319-E1325.

Hasinoff BB, Patel D, O'Hara KA (2008) Mechanisms of myocyte cytotoxicity induced by the multiple receptor tyrosine kinase inhibitor sunitinib. Mol Pharmacol 74: 1722-1728.

Jones AL, Barlow M, Barrett-Lee PJ, Canney PA, Gilmour IM, Robb SD, Plummer CJ, Wardley AM, Verrill MW (2009) Management of cardiac health in trastuzumab-treated patients with breast cancer: updated United Kingdom National Cancer Research Institute recommendations for monitoring. Br J Cancer 100: 684-692.

Johnston S, Pippen Jr J, Pivot X, Lichinitser M, Sadeghi S, Dieras V, Gomez HL, Romieu G, Manikhas A, Kennedy MJ, Press MF, Maltzman J, Florance A, O’Rourke L, Oliva C, Stein S, Pegram M (2009) Lapatinib combined with letrozole versus letrozole and placebo as first-line therapy for postmenopausal hormone receptor-positive metastatic breast cancer. $J$ Clin Oncol 27: 5538-5546.
Kerkelä R, Grazette L, Yacobi R, Iliescu C, Patten R, Beahm C, Walters B, Shevtsov S, Pesant S, Clubb FJ, Rosenzweig A, Salomon RN, Van Etten RA, Alroy J, Durand JB, Force T (2006) Cardiotoxicity of the cancer therapeutic agent imatinib mesylate. Nat Med 12: 908-916.

Kerkela R, Woulfe KC, Durand JB, Vagnozzi R, Kramer D, Chu TF, Beahm C, Chen MH, Force T (2009) Sunitinib-induced cardiotoxicity is mediated by off-target inhibition of AMP-activated protein kinase. Clin Transl Sci 2: $15-25$.

Khand AU, Shaw M, Gemmel I, Cleland JG (2005) Do discharge codes underestimate hospitalisation due to heart failure? Validation study of hospital discharge coding for heart failure. Eur J Heart Fail 7: 792-797.

Kim TD, le Coutre P, Schwarz M, Grille P, Levitin M, Fateh-Moghadam S, Giles FJ, Dörken B, Haverkamp W, Köhncke C (2012) Clinical cardiac safety profile of nilotinib. Haematologica 97: 883-889.

Krause DS, Van Etten RA (2005) Tyrosine kinases as targets for cancer therapy. N Engl J Med 353: 172-187.

Kümler T, Gislason GH, Kirk V, Bay M, Nielsen OW, Køber L, Torp-Pedersen C (2008) Accuracy of a heart failure diagnosis in administrative registers. Eur J Heart Fail 10(7): 658-660.

Larson RA, Hochhaus A, Hughes TP, Clark RE, Etienne G, Kim DW, Flinn IW, Kurokawa M, Moiraghi B, Yu R, Blakesley RE, Gallagher NJ, Saglio G, Kantarjian HM (2012) Nilotinib vs imatinib in patients with newly diagnosed Philadelphia chromosome-positive chronic myeloid leukemia in chronic phase: ENESTnd 3-year follow-up. Leukemia 26: 2197-2203.

Lee DS, Donovan L, Austin PC, Gong Y, Liu PP, Rouleau JL, Tu JV (2005) Comparison of coding of heart failure and comorbidities in administrative and clinical data for use in outcomes research. Med Care 43: 182-188.

Li A, Huang X, Song Y, Chen X, Sun J, Xu H, Wang Z (2015) Anti-epidermal growth factor receptor-targeted therapy in upper gastrointestinal tract cancers: a meta-analysis. Growth Factors 33: 113-127.

Mak IT, Kramer JH, Chmielinska JJ, Spurney CF, Weglicki WB (2015) EGFRTKI, erlotinib, causes hypomagnesemia, oxidative stress, and cardiac dysfunction: attenuation by NK-1 receptor blockade. J Cardiovasc Pharmacol 65: 54-61.

Martin M, Pienkowski T, Mackey J, Pawlicki M, Guastalla JP, Weaver C, Tomiak E, Al-Tweigeri T, Chap L, Juhos E, Guevin R, Howell A, Fornander T, Hainsworth J, Coleman R, Vinholes J, Modiano M, Pinter T, Tang SC, Colwell B, Prady C, Provencher L, Walde D, Rodriguez-Lescure A, Hugh J, Loret C, Rupin M, Blitz S, Jacobs P, Murawsky M, Riva A, Vogel C. Breast Cancer International Research Group 001 Investigators (2005) Adjuvant docetaxel for node-positive breast cancer. N Engl J Med 352: 2302-2313.

Marty M, Cognetti F, Maraninchi D, Snyder R, Mauriac L, Tubiana-Hulin M, Chan S, Grimes D, Antón A, Lluch A, Kennedy J, O’Byrne K, Conte P, Green M, Ward C, Mayne K, Extra JM (2005) Randomized phase II trial of the efficacy and safety of trastuzumab combined with docetaxel in patients with human epidermal growth factor receptor 2-positive metastatic breast cancer administered as first-line treatment: the M77001 study group. J Clin Oncol 23: 4265-4274.

Matok I, Gorodischer R, Koren G, Sheiner E, Wiznitzer A, Levy A (2009) The safety of metoclopramide use in the first trimester of pregnancy. $N$ Engl $J$ Med 360: 2528-2535.

Maxwell CB, Jenkins AT (2011) Drug-induced heart failure. Am J Health Syst Pharm 68: 1791-1804.

McArthur HL, Rugo H, Nulsen B, Hawks L, Grothusen J, Melisko M, Moasser M, Paulson M, Traina T, Patil S, Zhou Q, Steingart R, Dang C, Morrow M, Cordeiro P, Fornier M, Park J, Seidman A, Lake D,

Gilewski T, Theodoulou M, Modi S, D’Andrea G, Sklarin N, Robson M, Moynahan ME, Sugarman S, Sealey JE, Laragh JH, Merali C, Norton L, Hudis CA, Dickler MN (2011) A feasibility study of bevacizumab plus dose-dense doxorubicin-cyclophosphamide $(\mathrm{AC})$ followed by nanoparticle albumin-bound paclitaxel in early-stage breast cancer. Clin Cancer Res 17: $3398-4407$.

Moslehi JJ (2016) Cardiovascular toxic effects of targeted cancer therapies. N Engl J Med 375: 1457-1467.

Motzer RJ, Hutson TE, Tomczak P, Michaelson MD, Bukowski RM, Rixe O, Oudard S, Negrier S, Szczylik C, Kim ST, Chen I, Bycott PW, Baum CM, Figlin RA (2007) Sunitinib versus interferon alfa in metastatic renal-cell carcinoma. N Engl J Med 356: 115-124.

O’Brien SG, Guilhot F, Larson RA, Gathmann I, Baccarani M, Cervantes F, Cornelissen JJ, Fischer T, Hochhaus A, Hughes T, Lechner K, Nielsen JL, Rousselot P, Reiffers J, Saglio G, Shepherd J, Simonsson B, Gratwohl A, 
Goldman JM, Kantarjian H, Taylor K, Verhoef G, Bolton AE, Capdeville R, Druker BJ, IRIS Investigators (2003) Imatinib compared with interferon and low-dose cytarabine for newly diagnosed chronicphase chronic myeloid leukemia. N Engl J Med 348: 994-1004.

Ozcelik C, Erdmann B, Pilz B, Wettschureck N, Britsch S, Hübner N, Chien KR, Birchmeier C, Garratt AN (2002) Conditional mutation of the ErbB2 (HER2) receptor in cardiomyocytes leads to dilated cardiomyopathy. Proc Natl Acad Sci USA 99: 8880-8885.

Page 2nd RL, O'Bryant CL, Cheng D, Dow TJ, Ky B, Stein CM, Spencer AP, Trupp RJ, Lindenfeld J, American Heart Association Clinical Pharmacology and Heart Failure and Transplantation Committees of the Council on Clinical Cardiology; Council on Cardiovascular Surgery and Anesthesia; Council on Cardiovascular and Stroke Nursing; and Council on Quality of Care and Outcomes Research (2016) Drugs that may cause or exacerbate heart failure: a scientific statement from the American Heart Association. Circulation 134: e32-e69.

Perez EA, Suman VJ, Davidson NE, Sledge GW, Kaufman PA, Hudis CA, Martino S, Gralow JR, Dakhil SR, Ingle JN, Winer EP, Gelmon KA, Gersh BJ, Jaffe AS, Rodeheffer RJ (2008) Cardiac safety analysis of doxorubicin and cyclophosphamide followed by paclitaxel with or without trastuzumab in the North Central Cancer Treatment Group N9831 adjuvant breast cancer trial. J Clin Oncol 26: 1231-1238.

Quezado ZM, Wilson WH, Cunnion RE, Parker MM, Reda D, Bryant G, Ognibene FP (1993) High-dose ifosfamide is associated with severe, reversible cardiac dysfunction. Ann Intern Med 118: 31-36.

Radhakrishnan V, Bakhshi S (2011) 5-Fluorouracil-induced acute dilated cardiomyopathy in a pediatric patient. J Pediatr Hematol Oncol 33: 323.

Richardson DB. An incidence density sampling program for nested casecontrol analyses (2004) Occup Environ Med 61: e59.
Schneeweiss S, Suissa S (2012) Advanced approaches to controlling confounding in pharmacoepidemiologic studies. In Pharmacoepidemiology, Strom BL, Kimmel SE, Hennessy S (eds), pp 868-891. Wiley-Blackwell: Chichester, UK.

Seidman A, Hudis C, Pierri MK, Shak S, Paton V, Ashby M, Murphy M, Stewart SJ, Keefe D (2002) Cardiac dysfunction in the trastuzumab clinical trials experience. J Clin Oncol 20: 1215-1221.

Siegel R, DeSantis C, Virgo K, Stein K, Mariotto A, Smith T, Cooper D, Gansler T, Lerro C, Fedewa S, Lin C, Leach C, Cannady RS, Cho H, Scoppa S, Hachey M, Kirch R, Jemal A, Ward E (2012) Cancer treatment and survivorship statistics, 2012. CA Cancer J Clin 62: 220-241.

Stewart T, Pavlakis N, Ward M (2010) Cardiotoxicity with 5-fluorouracil and capecitabine: more than just vasospastic angina. Intern Med J 40: 303-307.

Vermorken JB, Mesia R, Rivera F, Remenar E, Kawecki A, Rottey S, Erfan J, Zabolotnyy D, Kienzer HR, Cupissol D, Peyrade F, Benasso M, Vynnychenko I, De Raucourt D, Bokemeyer C, Schueler A, Amellal N, Hitt R (2008) Platinum-based chemotherapy plus cetuximab in head and neck cancer. N Engl J Med 359: 1116-1127.

Yeh ET, Bickford CL (2009) Cardiovascular complications of cancer therapy: incidence, pathogenesis, diagnosis, and management. J Am Coll Cardiol 53: 2231-2247.

This work is published under the standard license to publish agreement. After 12 months the work will become freely available and the license terms will switch to a Creative Commons AttributionNonCommercial-Share Alike 4.0 Unported License.

Supplementary Information accompanies this paper on British Journal of Cancer website (http://www.nature.com/bjc) 